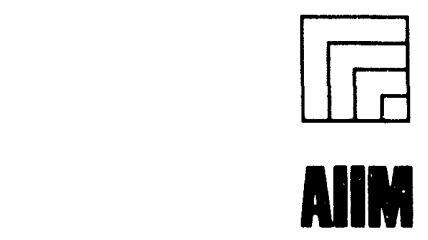

Association for Information and Image Management

1100 Wayne Avenue. Suite 1100

Silver Spring Maryiand 20910

$301 / 587.8202$

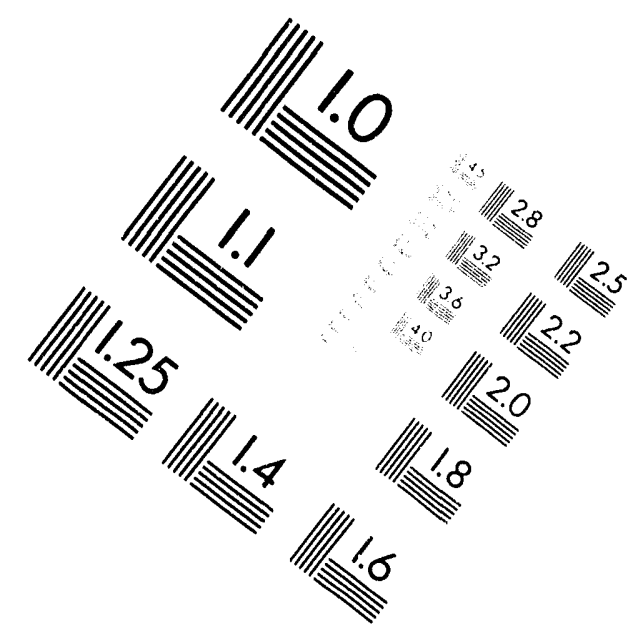

Centimeter

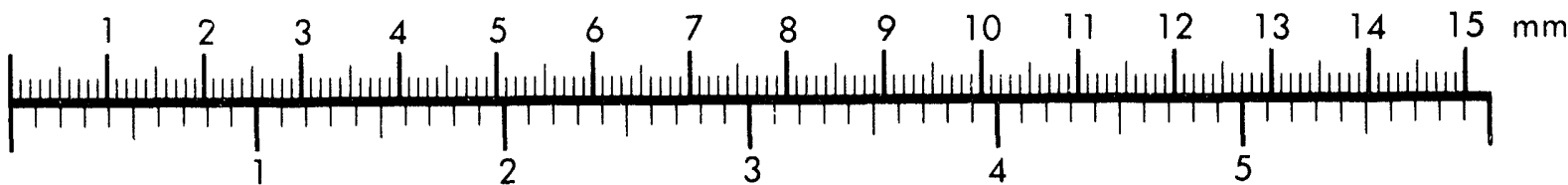

Inches
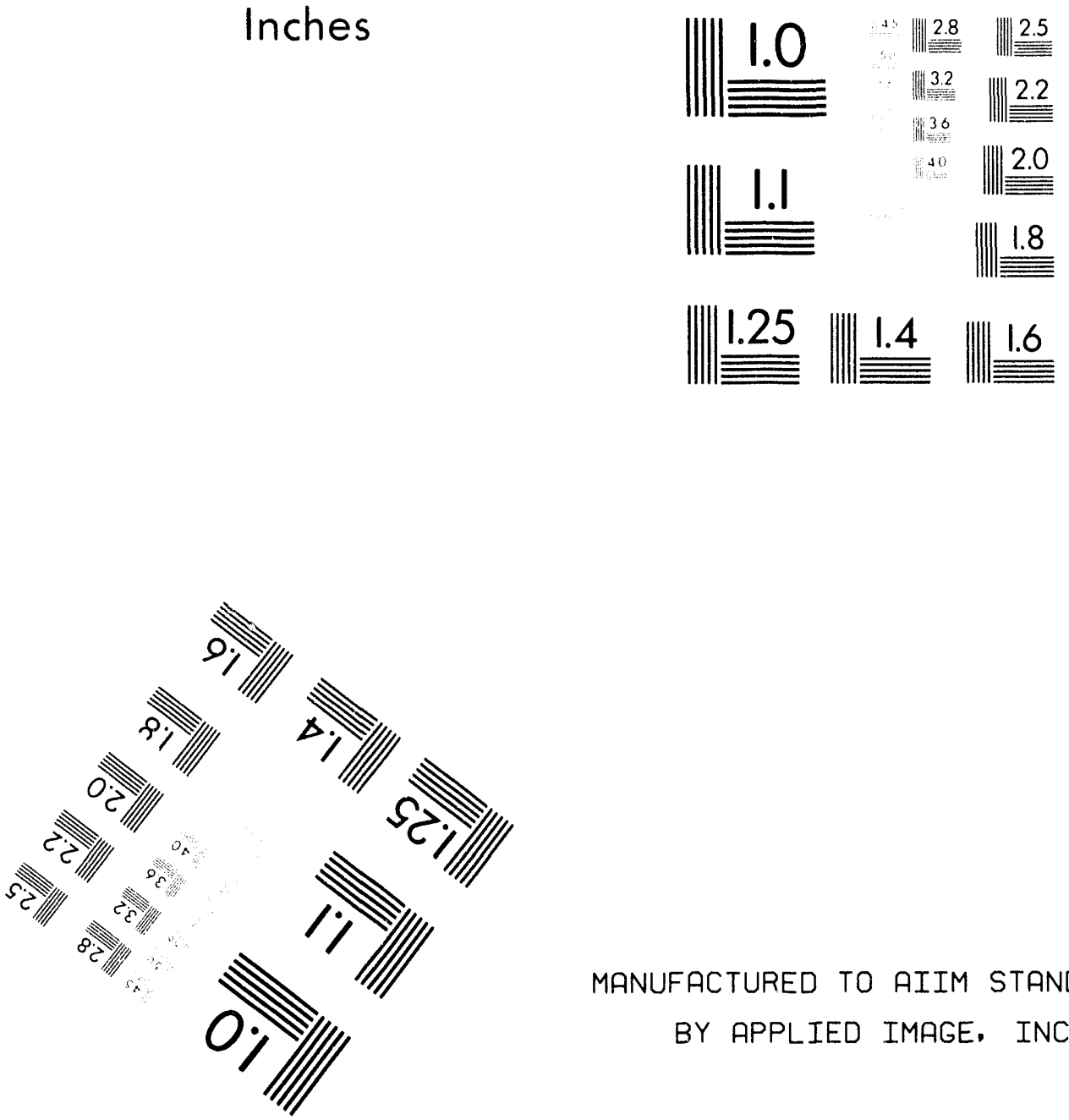

MANUFACTURED TO AIIM STANDARDS

BY APPLIED IMAGE, INC.

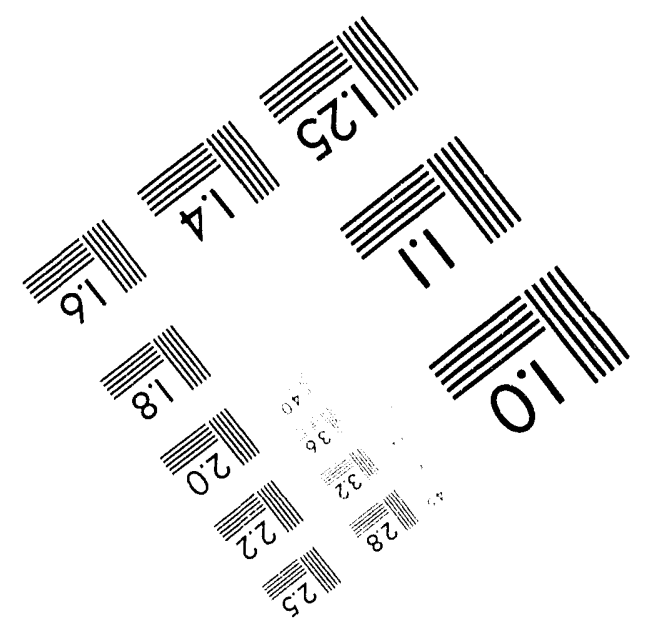




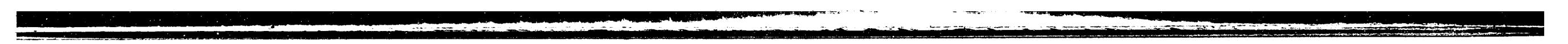
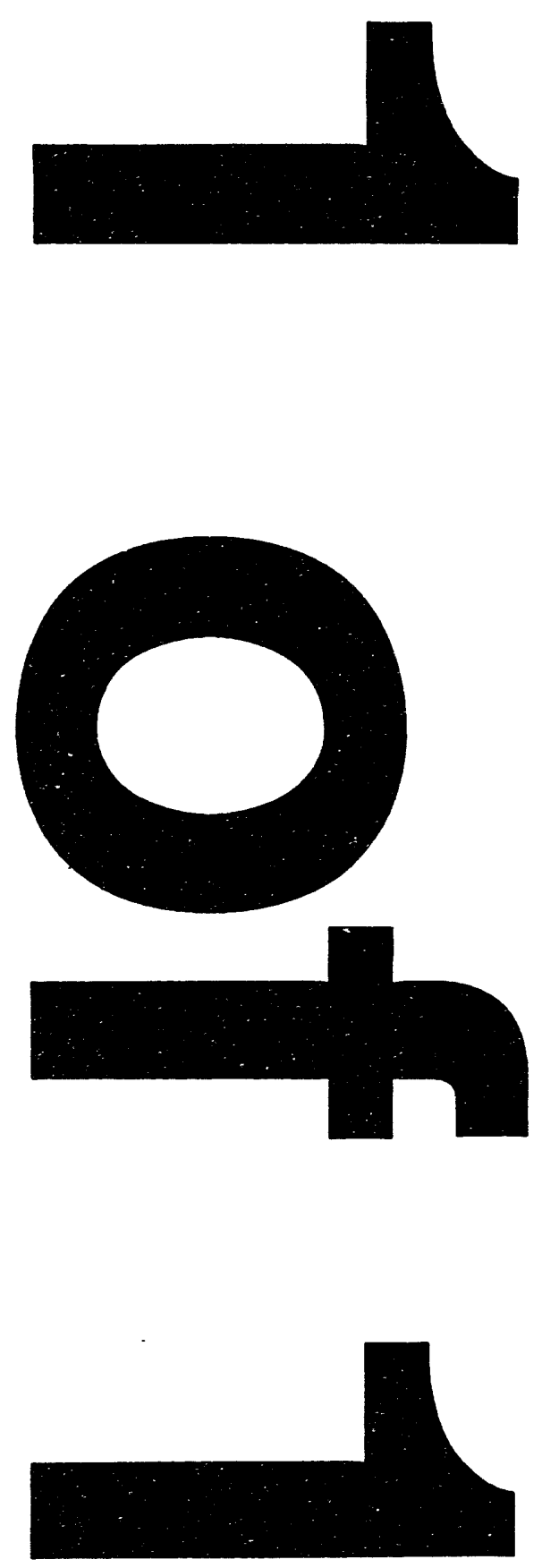

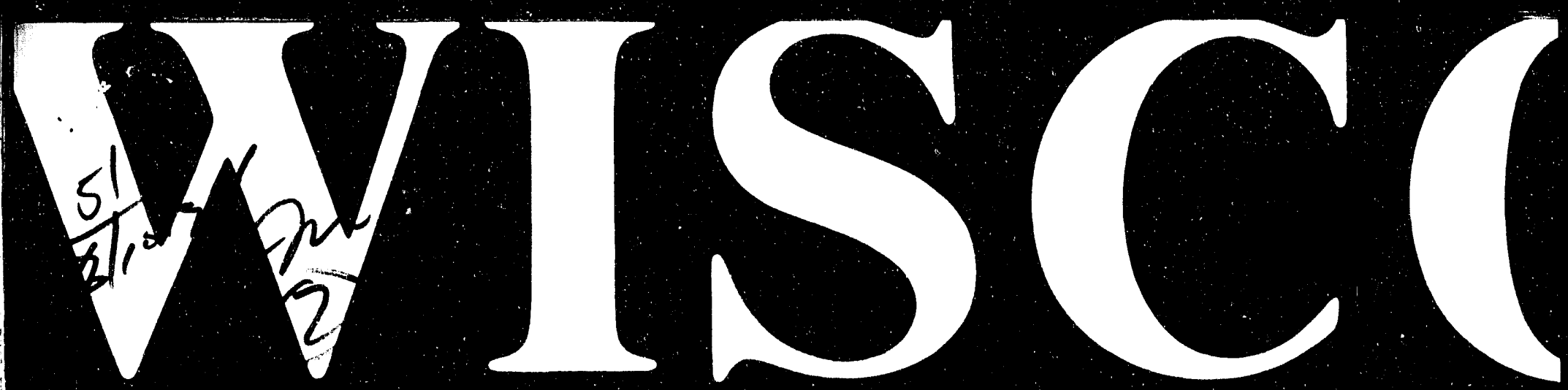

UNIVERSITY OF WISCONSIN • MADISON, WISCONSIN

\section{PLASMA PHYSICS}

\section{A fast spectroscopic diagnostic for the measurement of plasma impurity ion dynamics}

\section{D.J. Den Hartog \\ R.J. Fonck*}
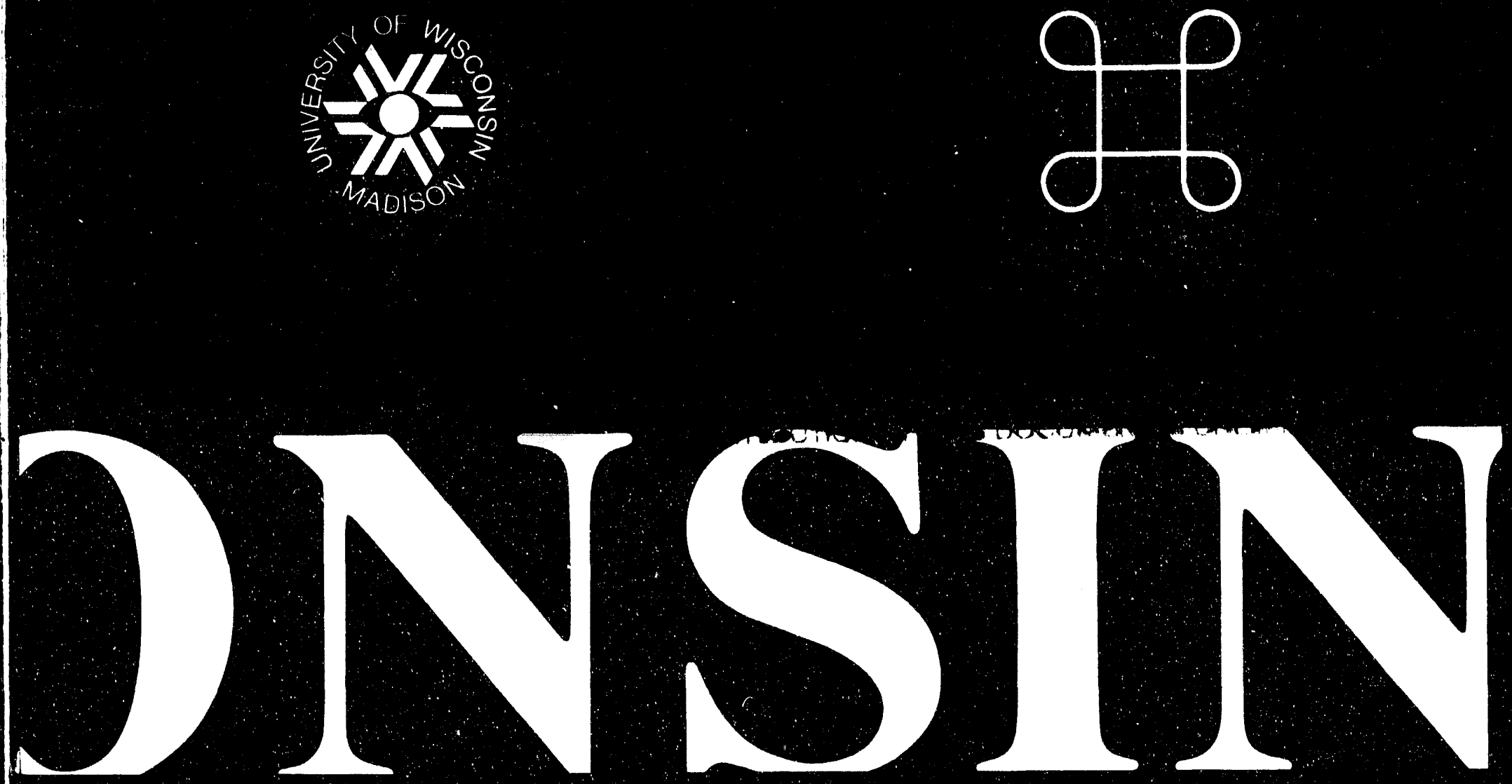


\section{NOTICE}

This report was prepared as an account of work sponsored by an agency of the United States Government. Neither the United States nor any agency thereof, nor any of their employees, makes any warranty, expressed or implied, or assumes any legal liability or responsibility for any third party's use or the results of such use of any information, apparatus, product or process disclosed in this report, or represents that its use by such third party would not infringe privately owned rights.

Printed in the United States of America Available from

National Technical Information Service

U.S. Department of Commerce

5285 Port Royal Road

Springfield, VA 22161

NTIS Price codes

Printed copy:

$\mathrm{A} 02$

Microfiche copy: 


\title{
A fast spectroscopic diagnostic for the measurement of plasma impurity ion dynamics
}

\section{J. Den Hartog}

University of Wisconsin-Madison, Department of Physics, Madison, Wisconsin 53706, U.S.A.

\section{R. J. Fonck}

University of Wisconsin-Madison, Department of Nuclear Engineering and Engineering Physics, Madison, Wisconsin 53706, U.S.A.

\begin{abstract}
A high-resolution spectrometer has been developed and used to measure simultaneously impurity ion temperatures and flow velocities in high temperature plasmas with $10 \mu \mathrm{s}$ temporal resolution (limited by digitization rate). This device is actually a duo-spectrometer: measurements from two different chordal views of the plasma can be made simultaneously via two separate quartz input fiber optic bundles coupled to the entrance slits which are tilted to compensate for line curvature. The dispersed spectra on the exit plane of the duo-spectrometer are coupled via quartz fiber optics to two arrays of 16 photomultiplier tubes each. Measurements made by recording the Doppler broadened and shifted $227.091 \mathrm{~nm}$ emission from the $C V$ impurity ions in the MST reversed-field pinch (RFP) plasma have achieved precisions of $<6 \mathrm{eV}$ for temperatures of $150 \mathrm{eV}$ and $<0.7 \mathrm{~km} / \mathrm{s}$ for flow velocities of $6 \mathrm{~km} / \mathrm{s}$. Representative results from the MST RFP indicate that the toroidal flow velocity drops and ion temperature increases during sawtooth events in MST.
\end{abstract}

PACS numbers: $52.70 . \mathrm{Kz}, 52.30 .-\mathrm{q}, 07.65 . \mathrm{Eh}, 42.81 . \mathrm{Wg}$

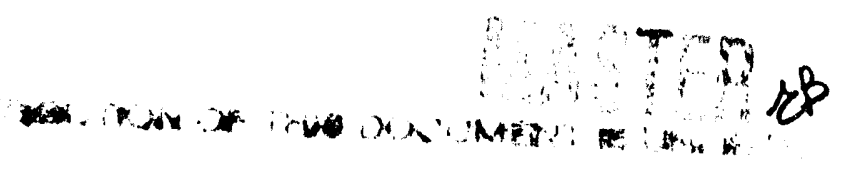




\section{INTRODUCTION}

\section{A. Purpose of diagnostic}

Measurement of the dynamics of the ions in a magnetically-confined plasma gives rich (and sometimes unexpected) insight into the physical principles that govern the behavior of the plasma as a whole. For example, a recent investigation in the MST RFP (reversed-field pinch) high-temperature plasma confinement device ${ }^{1}$ demonstrated that the majority ions are anomalously hot and that ion heating is correlated with discrete dynamo events. ${ }^{2}$ In tokamaks, knowledge of the local fluctuations in ion temperature and velocity is very useful to the development of an understanding of the mechanisms governing particle transport, ${ }^{3} \mathrm{H}$-mode operation, ${ }^{4}$ and the relationship between large-scale MHD phenomena such as sawtooth and locked modes and plasma flows and temperature. A quantity of particular interest in the RFP is the bulk plasma flow velocity because it has been postulated that correlated fluctuations in this velocity and the local magnetic field are responsible for the generation of the reversed toroidal magnetic field (a mechanism popularly known as the "MHD dynamo"). ${ }^{5}$ To explore this postulated behavior, we have developed a diagnostic system for high-speed measurements of plasma ion dynamics; this paper describes the design and performance of this system.

\section{B. Measurement technique}

The measurement technique is based on high-speed passive Doppler spectroscopy of intrinsic impurity ions in the MST plasma. The directed velocity of the ions is proportional to the Doppler wavelength shift of the line center; the temperature to the Doppler broadening of the line itself. The most convenient impurity line to which to apply this technique in MST is the $227.091 \mathrm{~nm}$ emission line of $\mathrm{CV}$, an ion which resides near the hot core of the MST plasma. Carbon is a common impurity in MST hydrogen plasmas, usually at an ion density of a few percent of the majority species. The $227.091 \mathrm{~nm}$ line is also attractive because it is one of the few reasonably bright lines from highly ionized impurity ions that is detectable without a vacuum spectrometer, and it consists of a single transition without complex fine structure. The relatively poor spatial resolution inherent in the use of passive spectroscopy with chordal views of the plasma poses no limitation to the present work on MST because the MHD fluctuations of interest are large-scale, with wavelengths on the order of the machine size. Improved spatial resolution can be readily achieved by applying this diagnostic system to active techniques such as charge-exchange recombination spectroscopy. 6,7 
Measurements of fluctuations in the spectral line center and width require an instrument with both high time resolution and a high luminosity-resolution product. The frequency spectrum of the velocity fluctuations is expected to peak around $20-30 \mathrm{kHz}$ in the lab frame since that is the range in which much of the observed MHD activity takes place in MST. 8 Thus, a minimal sampling frequency of $\sim 100 \mathrm{kHz}$ is required. High spectral resolution is required to observe values of $\widetilde{v} \sim 1 \mathrm{~km} / \mathrm{sec}$, a value suggested by 3-D MHD simulations of the velocity turbulence. ${ }^{9}$ This requires an ability to detect line shifts of $\sim 10^{-3} \mathrm{~nm}$. High luminosity is required to minimize photon noise in the measurements and optimize accuracy of the $\tilde{v}$ and $\tilde{T}$ measurements. Absent quantitative measures of the impurity emission levels from MST, this system was designed to optimize the étendue of a commercially available grating spectrometer, with modifications as described below.

\section{Other systems}

Passive Doppler spectroscopy has been applied to measure the dynamics of the impurity ions in many high-temperature plasmas. A typical approach employs an intensified linear photodiode array at the exit plane iri a highly dispersive spectrometer. While this type of system has good sensitivity and resolution, typical readout times are $\geq 1 \mathrm{~ms}$, although instruments developed for the DIII-D tokamak have reduced this time to $260 \mu \mathrm{s} .10,11,12$

For higher time resolution, multiple discrete detectors are used to record several spectral elements simultaneously at high sampling rates. There have been several instruments built with this capability, 13,14 including a UV/visible polychromator for the ZT-1 toroidal pinch 15 and a VUV polychromator for the ZT-4OM RFP. ${ }^{16}$ To date, the Doppler plasma diagnostic with the best combination of speed and accuracy appears to be the instrument on the COMPASS-C tokamak. ${ }^{17}$ This spectrometer uses three photomultipliers to simultaneously record three wavelength bands. The Doppler broadened spectral line is dispersed such that it is divided into these three roughly equal wavelength regions. Precisions of $\sim 1 \mathrm{~km} / \mathrm{s}$ were achieved with $40 \mu$ s time resolution.

\section{The MST system}

The system implemented on MST and described in this paper was designed to extend the approach used on COMPASS-C in two respects: 1) the system etendue is optimized by using tall entrance slits combined with a simple aberration correction to retain high spectral resolution; 
and 2) the number of spectral channels is expanded considerably (to $\geq 8$ channels across the line profile) to decouple small fluctuations in the line centroid from those in the line width. Our instrument simultaneously records two separate chordal views of the plasma, each with 16 individual wavelength channels (this capability makes it a duo-spectrometer). This system combines good étendue with the simplicity and flexibility of a fiber-optically-coupled singlegrating spectrometer.

\section{APPARATUS}

\section{A. Optical design}

Ray tracing was performed to maximize the luminosity-resolution product achievable with the available monochromator, an $f / 10$ Czerny-Turner design with $1.0 \mathrm{~m}$ focal length (Jarrell-Ash). The duo-spectrometer design was optimized with the constraint that the basic structure (mirrors, mirror mounts, grating mount and drive) remain unmodified and that the instrumental linewidth be $\Delta \lambda \approx 0.025 \mathrm{~nm}$, as discussed below. This left the entrance slit and exit plane configurations and the grating itself as the major variables; these were configured using the BEAM FOUR software package from Stellar Software.

The dominant aberration in our instrument is line curvature, 18,19,20 which is caused by the variation in dispersion that occurs as a function of slit height. For our Czerny-Turner monochromator, a straight vertical slit on the entrance plane maps to an ellipse on the exit plane (or vice versa). As long as the slit is fairly wide, such line curvature can be compensated quite well by a simple tilt of a straight slit. This configuration is illustrated in Fig. 1(a), which shows the two entrance slits, one above and the other below the optical axis of the spectrometer. The top of the upper slit (and the bottom of the lower slit) are tilted $0.5^{\circ}$ toward the exit plane array (Fig. 1(c)). This tilt, although small, reduces the width of the exit plane image by about $40 \%$ compared to a simple vertical entrance slit. Slit height was limited because longer slits would have required curvature rather than just the simple tilt of a straight slit. (This type of compensation has been done, ${ }^{21}$ but was not implemented on our instrument due to cost limitations.) Figure 1(b) shows the expected exit plane images; they are welldefined vertical rectangles that exhibit a small amount of anamorphic magnification. Note that astigmatic blurring is small and does not lead to vertical overlap of the two images.

A simulation was performed to assure that the duo-spectrometer configuration provided the resolution necessary to measure flow velocities to accuracies of less than $1 \mathrm{~km} / \mathrm{sec}$ (with 
a)

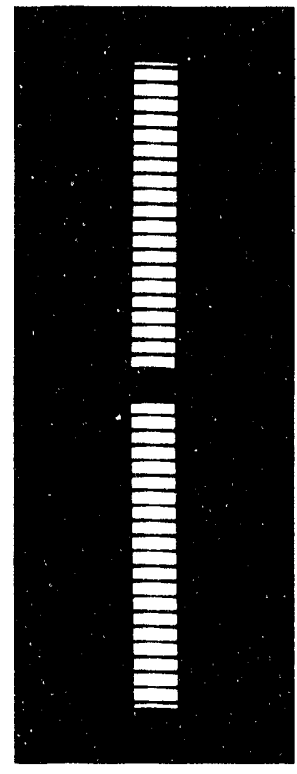

b)

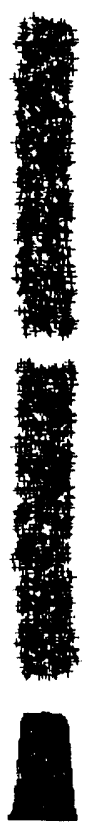

c)

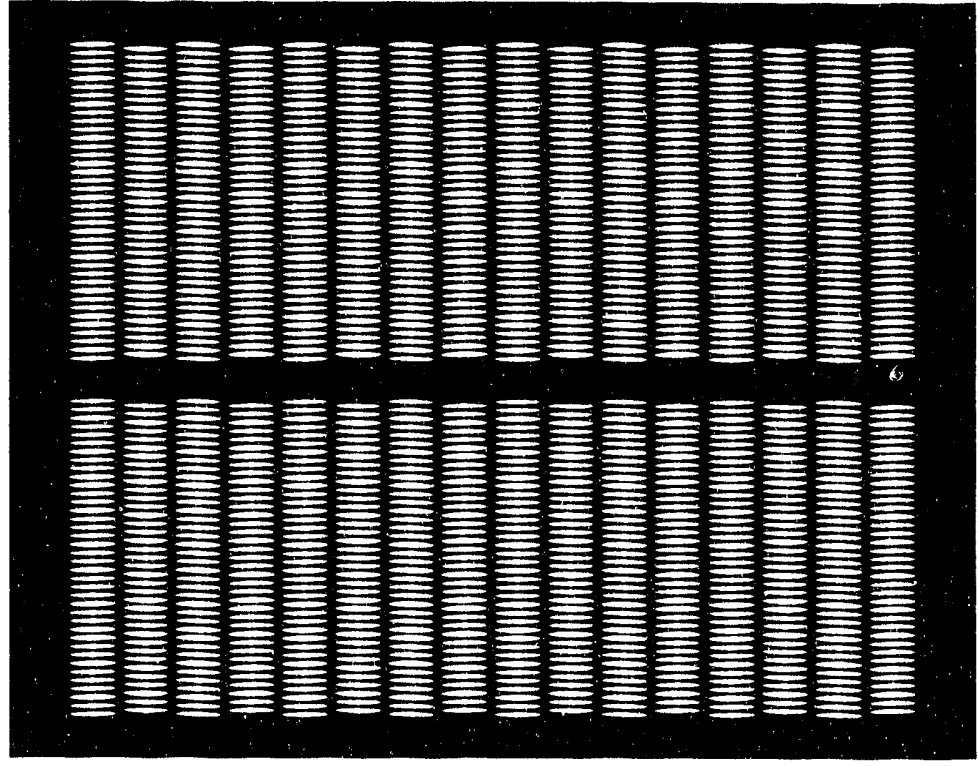

Scales:

$1.0 \mathrm{~mm} \mathrm{I}$

FIG. 1. (a) The entrance slits consisting of a mask over the fiber optic arrays, (b) the ray-traced exit plane images of the entrance slits, and (c) the exit plane fiber optic arrays. Note the different horizontal and vertical scales. 
$50 \leq T_{i} \leq 1000 \mathrm{eV}$ ). Our aim was to maximize the étendue of the duo spectrometer by using the widest slits consistent with this accuracy requirement. We wrote an IDL computer program which simulated the detection characteristics of the duo-spectrometer. This simulation program allowed variation of the width and shape of the wavelength channels (corresponding to slit width and instrument function) and the total number of wavelength detection channels. The parameters of interest (velocity and temperature) were reconstructed from the simulated spectra while varying the instrument parameters. The accuracy with which these reconstructed parameters matched the program input velocity and temperature provided a figure of merit.

Lacking accurate intensity estimates, we arbitrarily and conservatively estimated that the signal would be barely above the photon counting level, i.e., each timestep would require reconstruction of the velocity and temperature from a Doppler broadened spectrum defined by only a few hundred counts. Even at the low signal levels of the simulation Doppler shifts that were a few percent of the channel widths (in $\mathrm{nm}$ ) could be resolved. The number of wavelength channels was determined by the range of ion temperatures to be measured. For typical MST ion temperatures we required that half of the 16 available channels contain measurable signal; this allowed substantial flexibility as only three illuminated channels are needed to define the Doppler broadened spectrum. The final design parameters chosen were these: input slit width = $178 \mu \mathrm{m}$ and exi. slit width $=200 \mu \mathrm{m}$, giving an instrumental line width of $0.0246 \mathrm{~nm}$.

\section{B. Duo-spectrometer}

The spectrometer itself is a standard $f / 101.0 \mathrm{~m}$ focal length Czerny-Turner configuration. The mirrors and grating were overcoated with $\mathrm{MgF}_{2}$ to enhance UV reflectivity. The $1180 \mathrm{~g} / \mathrm{mm}$ grating is blazed at $1000 \mathrm{~nm}$ in first order, giving the desired $0.12 \mathrm{~nm} / \mathrm{mm}$ reciprocal dispersion in fifth order for $227 \mathrm{~nm}$.

The two entrance slits of the duo-spectrometer simply act as masks for the input fiber optic bundles. They were cut into a single piece of $12.7 \mu \mathrm{m}$ (0.0005 in.) thick stainless steel shim stock with a $178 \mu \mathrm{m}$ (0.007 in.) wide jeweler's saw. This slit mask is mounted on the front of the fixture that holds the two entrance fiber optic bundles. The fibers in each bundle are arranged in a vertical line behind the slit in the mask. (The slits in the mask were tilted at $0.5^{\circ}$ off vertical rather than tilting the fiber lines off vertical.) Alignment was done by filling the fiber optic bundles with light and positioning the slit mask while observing the assembly 
with a microscope.

The entrance fiber optic assembly was manufactured by $C$ Technologies (Verona, New Jersey). The assembly consists of two separate fiber optic bundles, one for each slit of the duospectrometer. The fixtures at the light collection end of each bundle hold the fibers in a round bundle. The individual fibers are $400 \mu \mathrm{m}$ diameter pure fused silica core with a doped fused silica cladding. The fiber jackets were removed in the slit fixture to enhance the packing efficiency in the line assembly. The manufacturer specified fiber attenuation of less than 1.0 $\mathrm{dB} / \mathrm{m}$ for $225 \mathrm{~nm}$ light. Each bundle was three meters long and encased in a PVC/monocoil sheath.

The fiber optic array (also manufactured by C Technologies) on the exit plane of the duo-spectrometer is more complex than the entrance slit assembly. In Fig. 1(c) is a face-on view of the array as packed in its aluminium fixture; this shows the large number of fibers in each of the two detector arrays. Each of the two arrays contains 16 vertical columns with each column containing 37 or 38 fibers. Note that each column is meshed with its neighbor such that the packing efficiency is maximized. Individual fibers consist of $200 \mu \mathrm{m}$ diameter pure fused silica core with a doped fused silica cladding. The polyamide jackets on the fibers were left intact even though doing so reduced the packing efficiency; removal of the jackets prior to fabrication of the assembly would have greatly increased the chance of "dead" (broken) fibers and the fabrication cost.

The anamorphic image of one of the entrance slits on the exit plane array is a vertical rectangle with a $200 \mu \mathrm{m}$ width that is well-matched to the core diameter of the individual fibers. The instrument functions were measured by passing the $226.502 \mathrm{~nm} \mathrm{Cd}$ II line (from a small cadmium low-temperature discharge lamp) over the exit plane array of the duospectrometer. (Particularly useful for absolute calibration of the wavelength scale is the fact that the fifth order of the $226.502 \mathrm{~nm}$ line and the fourth order of the $283.691 \mathrm{~nm}$ Cd I line nearly overlap.) Figure 2 is a graph of the instrument functions of two adjacent wavelength channels of the duo-spectrometer. These instrument functions are typical; note the lack of channel-to-channel crosstalk and the good approximation of the expected triangular instrument function.

Each individual vertical column in the exit-plane fiber optic array leaves the aluminium fixture as a separate bundle in a $\mathrm{PVC} /$ monocoil sheath. Each bundle is $0.5 \mathrm{~m}$ long and is terminated as a round bundle in a $6.35 \mathrm{~mm}(0.250 \mathrm{in}$.) diameter cylindrical aluminium 


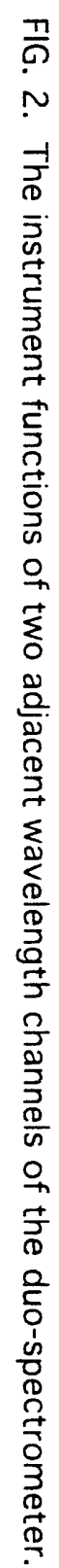

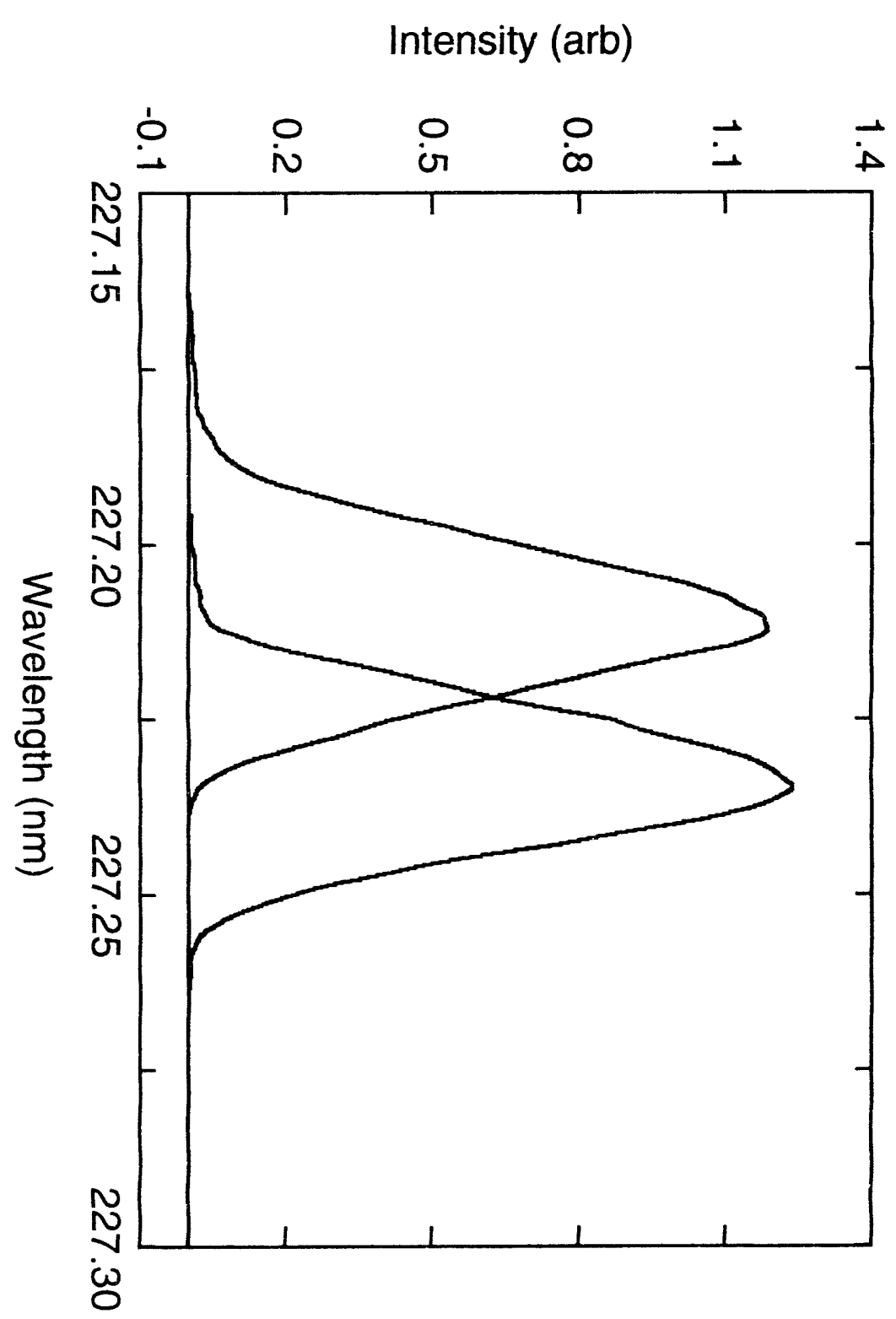


fixture. This is the face of the fiber that delivers light to photocathodes of the photomultiplier tubes (PMT). The cylindrical fixtures are held by simple o-ring compression fittings such that the face of the fiber is several $\mathrm{cm}$ from the photocathode of the THORN EMI type 9125QB quartz window PMT. This allows light to spread out in an $f / 10$ cone upon exit from the fiber face before it reaches the photocathode, which is $23 \mathrm{~mm}$ in diameter. Each of the 32 PMT's is individually shielded in a concentric assembly of high permeability iron surrounded by a soft iron tube with a wall thickness of $6.35 \mathrm{~mm}(0.250 \mathrm{in}$.). The 32 PMT's are mounted in a large array such that each tube is roughly co-linear with a radial line originating at the exit plane fiber array; this simplifies connection of each individual filer bundle to its proper PMT. The signal from each PMT goes to small card-mounted current-to-voltage amplifier. Each channel is independently digitized at $100 \mathrm{kHz}$ with 12-bit accuracy. Anti-aliasing filters (8-pole Butterworth with $f_{3 d B}=35 \mathrm{kHz}$ ) are used to condition the signal just prior to the digitizer input. These filters are also card-mounted and use the MAX274 continuous-time active filter integrated circuit.

\section{Light collection}

An overview of the diagnostic apparatus is shown is Fig. 3. The two chords of the light collection system view roughly the same emission volume of the plasma, but from opposite directions. Thus, for a given directed $\mathrm{Jw}$ velocity, one chord will collect line emission light Doppler shifted to a shorter wavelength, he other chord light shifted to a longer wavelength. Since the magnitude of the Doppler shift is equivalent for both directions, the exact position of the $227.091 \mathrm{~nm}$ line on the exit plane of the duo-spectrometer need not be determined prior to taking actual data on the ion dynamics of the plasma. The Doppler shift is simply half of the wavelength separation of the two shifted line centers. This is convenient because it is much easier to perform a relative wavelength calibration (channel-to-channel separation and channel half-width) than an absolute wavelength calibration (especially at the accuracies that would be required to measure a Doppler shift $\leq 1 \mathrm{~km} / \mathrm{s}$ ).

As can be seen in Fig. 3, each light collection chord is conical in shape. The collection face of each fiber bundle is focused on the surface of the opposing collection lens to minimize the amount of reflected light that is collected. The interference filters just in front of the collection faces of the fiber optics keep lower order UV and visible lines from interfering with the CV line in fifth order in the duo-spectrometer. 


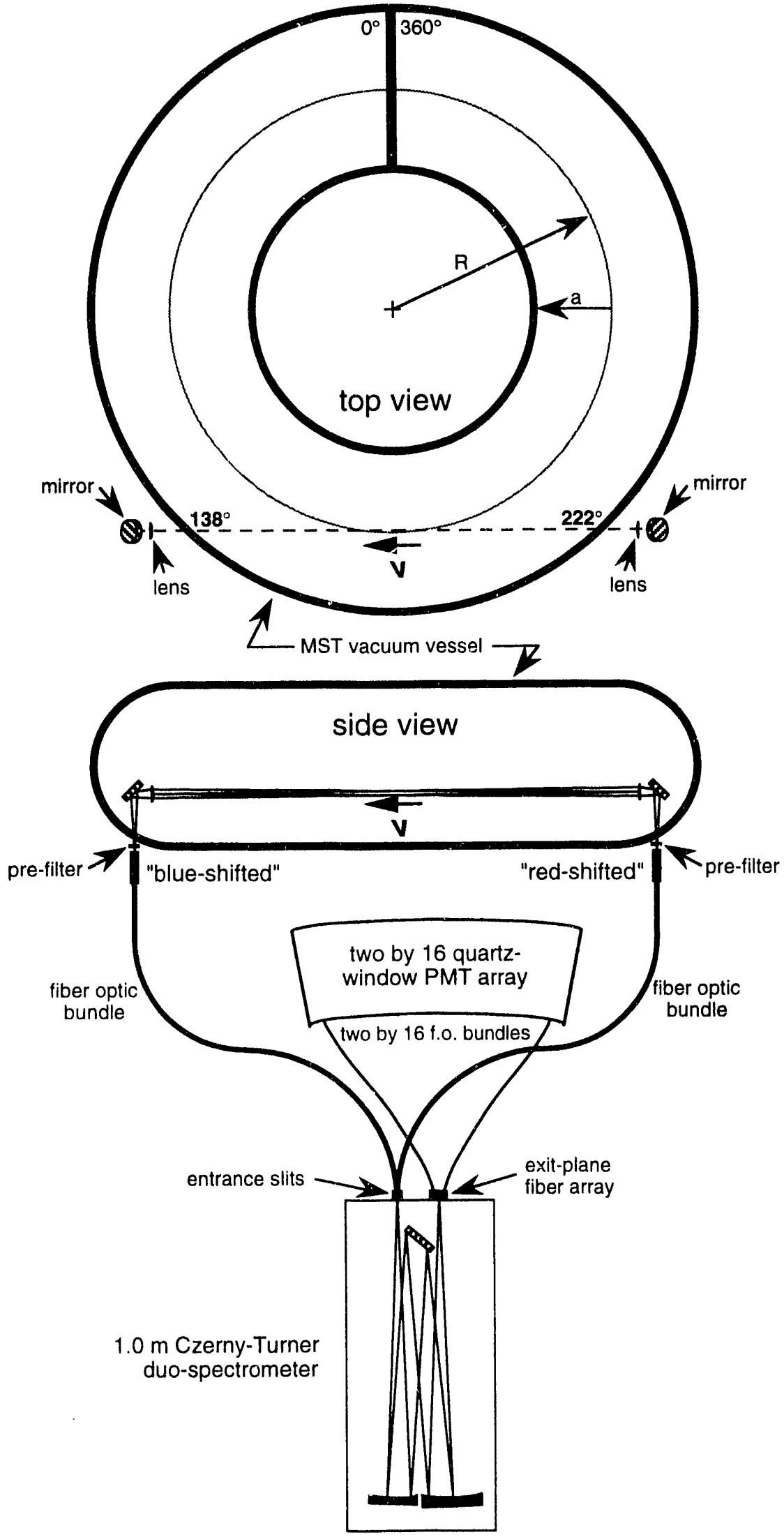

FIG. 3. An overview of the impurity ion dynamics diagnostic as installed on the MST RFP. Both a top view and side view of the MST vacuum vessel are shown. 
Limited spatial localization is acceptable for experiments such as ours in which we are observing phenomena that fluctuate over MHD scale lengths of about one meter. For high plasma current MST discharges, most of the $227.091 \mathrm{~nm}$ line emission originates from the plasma volume between $r / a=0.3$ and 0.7 .22 (The variable $r$ indicates the position along the minor radius of the toroid, $a$ is the minor radius which for MST is $0.52 \mathrm{~m}$.) This implies that our diagnostic is collecting light over this entire volume during high current discharges, resulting in a line-of-sight spatial average of about one meter. For low plasma current discharges, which have a lower electron temperature, the CV emission volume is probably nearer to the major axis of the toroid. Thus the tangential intersection of the light collection chords and the emission volume is smaller (see Fig. 3 ) and the spatial resolution is improved.

\section{RESULTS}

Reduction of the multiple measured waveforms to time records of ion temperature and flow velocity is done with a nonlinear least squares fitting routine. The IDL implementation of CURFIT $^{23}$ is used to fit a Gaussian function to the raw data. Figure 4 shows a typical result. The rms fluctuation level (which includes both plasma and noise fluctuations) between 10 and 11 $\mathrm{ms}$ is $<6 \mathrm{eV}$ for temperature and $<0.7 \mathrm{~km} / \mathrm{s}$ for flow velocity. Note the sudden increase in temperature and decrease in flow velocity that occurs during a sawtooth event.

The data shown in this paper are the result of initial operation of this diagnostic. Several upgrades are currently being made to this instrument. The replacement of the interference filter pre-filters (Fig. 3) with small concave holographic grating monochromators has the largest potential for positive impact. These monochromators will have $\geq 50 \%$ transmission at $227 \mathrm{~nm}$ (versus the $\leq 10 \%$ transmission of the interference filters) combined with a narrower passband and better rejection. This improvement should allow measurment of flow velocity to sub $\mathrm{km} / \mathrm{sec}$ accuracy. Also, the $100 \mathrm{kHz}$ digitizers currently being used will be replaced with $1 \mathrm{MHz}$ models to increase the fluctuation measurement bandwidth to $250 \mathrm{kHz}$.

\section{ACKNOWLEDGEMENTS}

The authors thank Abdulgader Almagri, Robert Ashley, Greg Garstka, Roch Kendrick, and Christopher Watts for contributing to the design, construction, and operation of this diagnostic.

This work was supported by the U. S. Department of Energy. 

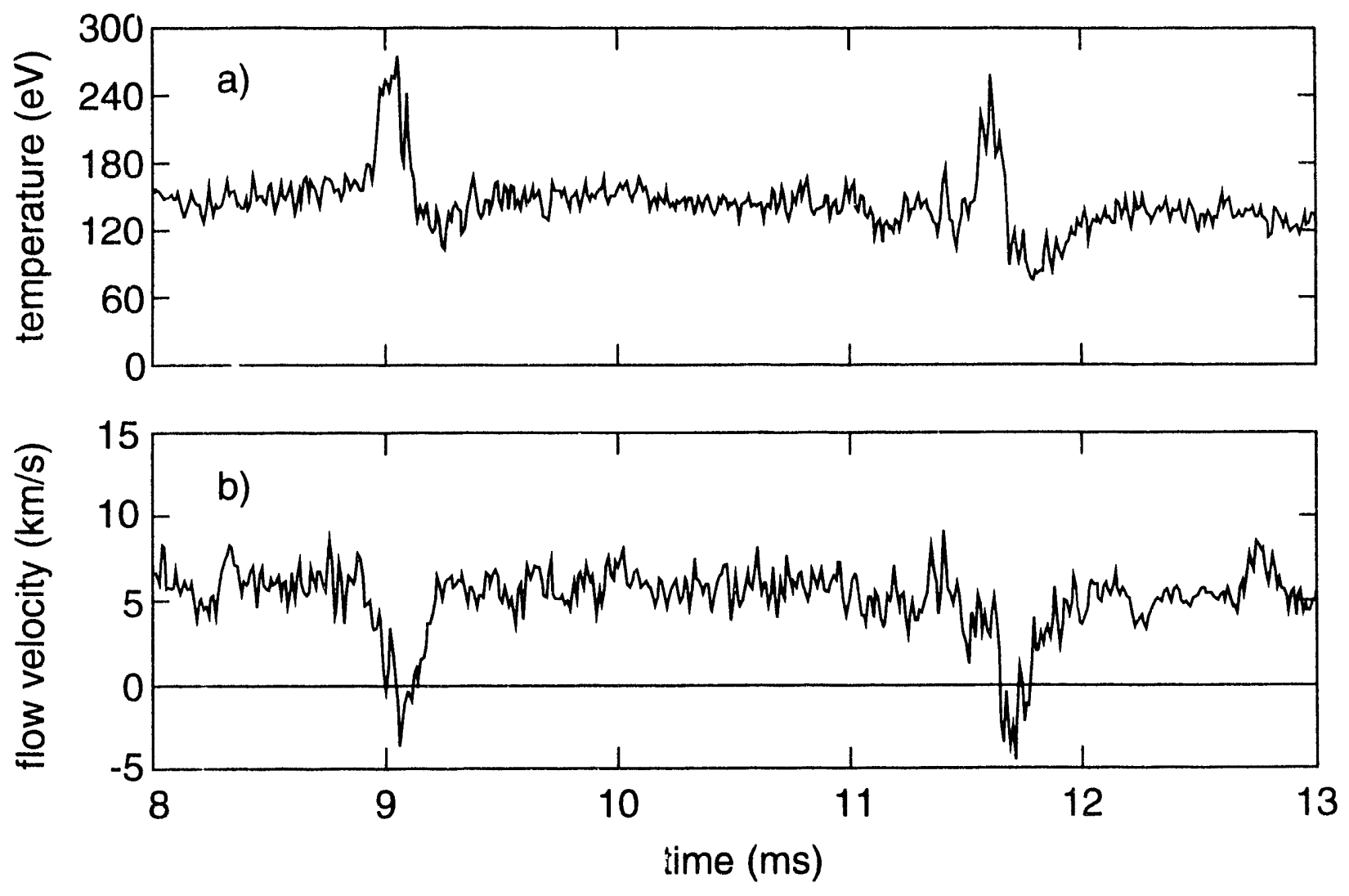

FIG. 4. The (a) ion temperature and (b) flow velocity measured every $10 \mu \mathrm{s}$. 


\section{REFERENCES}

${ }^{1}$ R. N. Dexter, D. W. Kerst, T. W. Lovell, S. C. Prager, and J. C. Sprott, Fusion Technol. 19, 131 (1991).

2Earl Scime, Miodrag Cekic, D. J. Den Hartog, Samuel Hokin, D. J. Holly, and Christopher Watts, Phys. Fluids B 44062 (1992).

${ }^{3}$ H. Biglari, P. H. Diamond, and P. W. Terry, Phys. Fluids 31, 2644 (1988).

4R. J. Groebner, K. H. Burrell, and R. P. Seraydarian, Phys. Rev. Lett. 64, 3015 (1990).

${ }^{5}$ Sergio Ortolani and Dalton D. Schnack, Magnetohydrodynamics of Plasma Relaxation, (World Scientific, Singapore, 1993), p. 95.

${ }^{6}$ R. J. Fonck, D. S. Darrow, and K. P. Jaehnig, Phys. Rev. A 29, 3288 (1984).

${ }^{7}$ A. Boileau, M. von Hellermann, L. D. Horton, J. Spence, and H. P. Summers, Plasma Phys. Contr. Fusion 31, 779 (1989).

${ }^{8}$ T. D. Rempel, A. F. Almagri, S. Assadi, D. J. Den Hartog, S. A. Hokin, S. C. Prager, J. S. Sarff, W. Shen, K. L. Sidikman, C. W. Spragins, J. C. Sprott, M. R. Stoneking, and E. J. Zita, Phys. Fluids B 4, 2136 (1992).

${ }^{9}$ Yung-Lung Ho, Ph.D. thesis, University of Wisconsin-Madison, 1988.

${ }^{10}$ R. P. Seraydarian, K. H. Burrell, N. H. Brooks, R. J. Groebner, and C. Kahn, Rev. Sci. Instrum. 57, 155 (1986).

${ }^{11}$ Raymond P. Seraydarian and Keith H. Burrell, Rev. Sci. Instrum. 57, 2012 (1986).

${ }^{12}$ P. Gohil, K. H. Burrell, R. J. Groebner, and R. P. Seraydarian, Rev. Sci. Instrum. 61, 
2949 (1990).

${ }^{13}$ M. G. Bell, Plasma Phys. 19, 467 (1977); Nucl. Fusion 19, 33 (1979).

${ }^{14}$ G. E. Georgiou, T. C. Marshall, and P. G. Weber, Phys. Fluids 23, 2085 (1980).

${ }^{15}$ R. B. Howell and H. J. Karr, Phys. Fluids 19, 2012 (1976).

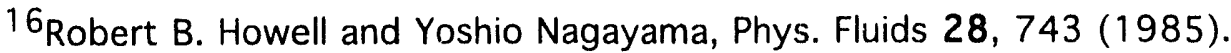

${ }^{17}$ R. A. Bamford, P. G. Carolan, and C. A. Bunting, Rev. Sci. Instrum. 63, 4962 (1992).

${ }^{18}$ Keiei Kudo, Science of Light 9, 1 (1960).

19 M. V. R. K. Murty, Optical Engineering 13, 23 (1974).

${ }^{20}$ Arthur B. Shafer, Lawrence R. Megill, and LeAnn Droppleman, J. Opt. Soc. Am. 54, 879 (1964).

${ }^{21}$ T. Thorson, G. McKee, R. J. Fonck, and B. Stratton, Rev. Sci. Instrum. 63, 5182 (1992).

${ }^{22}$ Christopher Watts, “Doppler Broadened Impurity Ion Temperatures, Radial Emission and Temperature Profiles in the MST," University of Wisconsin-Madison, Plasma Physics PLP 1118,1993 (unpublished).

${ }^{23}$ Philip R. Bevington, Data Reduction and Error Analysis for the Physical Sciences (McGraw-Hill, New York, 1969), p. 237. 
S.N. Rasband, Brigham Young University

T. Dolan, EG\&G Idaho, Inc.

R.A. Moyer, General Atomics

J.B. Taylor, Institute for Fusion Studies, The University of Texas at Austin

E. Uchimoto, University of Montana

F.W. Perkins, PPPL

O. Ishihara, Texas Technical University

M.A. Abdou, University of California, Los Angeles

R.W. Conn, University of California, Los Angeles

P.E. Vandenplas, Association Euratom-Etat Belge, Belgium

Centro Brasileiro de Pesquisas Firicas, Brazil

P. Sakanaka, Institute de Fisica-Unicamp, Brazil

Mme. Monique Bex, GANIL, France

J. Radet, CEN/CADARACHE, France

University of Ioannina, Greece

S. Ortolani, Istituto Gas Ionizzati, EURATON-ENEA-CNR Association, Italy

R. Andreani, Associazione EURATOM-ENEA sulla Fusione, Italy

Plasma section, Energy Fundamentals Division Electrotechnical Laboratory, Japan

Y. Kondoh, Gunma University, Kiryu, Gunma, Japan

H. Toyama, University of Tokyo, Japan

Z. Ypsjoda. University of Tokyo, Japan

FOM-Instituut voor Plasmafysica "Rijnhuizen," The Netherlands

Z. Ning, Academia Sinica, Peoples Republic of China

P. Yang, Shandong University, Peoples Republic of China

S. Zhu, University of Science \& Technology of China, People's Republic of China

I.N. Bogatu, Institute of Atomic Physics, Romania

M.J. Alport, University of Natal, Durban, South Africa

R. Storer, The Flinders University of South Australia, South Australia

B. Lehnert, Royal Institute of Technology, Sweden

Librarian, CRPP, Ecole Polytechnique Federale de Lausanne, Switzerland

B. Alper, Culham Laboratory, UK

A. Newton, UK

2 for Chicago Operations Office

5 for individuals in Washington Offices

INTERNAL DISTRIBUTION IN ADDITION TO UC-20

80 for local group and file 
